\title{
MSCs-derived exosomes attenuate ischemia- reperfusion brain injury and inhibit microglia apoptosis via exosomal miR-26a-5p mediated suppression of CDK6
}

\section{Chang Cheng}

xinqiao hospital third military medical university

\section{Xiuying Chen}

xinqiao hospital third military medical university

\section{Yuhan Wang}

xinqiao hospital third military medical university

\section{Wenchao Cheng}

xinqiao hospital third military medical university

\section{Xuzheng Zuo}

xinqiao hospital thirf military medical university

\section{Weiju Tang}

xinqiao hospital third military medical university

Wen Huang ( $\triangle$ WenHuangChongqing@163.com )

Xinqiao Hospital third military medical university https://orcid.org/0000-0002-0822-2690

Research article

Keywords: Exosomes, Mesenchymal stromal cells, miR-26a-5p, CDK6, ischemia-reperfusion injury

Posted Date: November 6th, 2020

DOI: https://doi.org/10.21203/rs.3.rs-100097/v1

License: (c) (i) This work is licensed under a Creative Commons Attribution 4.0 International License. Read Full License 


\section{Abstract}

Background: This study aims to explore the role of mesenchymal stromal cells (MSCs)-derived exosomes (MSCs-Exo) in the cerebral ischemia-reperfusion (I/R) injury.

Methods: Exosomes were isolated from MSCs of adult C57BL/ $6 \mathrm{~J}$ mice by the gradient centrifugation method. The expression of miR-26a-5p and CDK6 in MSCs-Exo and mice brain tissues were evaluated by qRT-PCR and western blot. MiR-26a-5p mimics and miR-NC were transfected into MSCs, and exosomes were isolated from the stably expressing MSCs. Then MSCs-Exo-miR-26a-5p mimics or MSCs-Exo-miR-NC was injected into mice through the tail vein, or added into medium to stimulate BV-2 cells. Cell viability was evaluated by CCK-8 assay. Cell apoptosis was detected by flow cytometry. The apoptosis in brain tissues was evaluated by TUNEL staining assay. Bioinformatics analysis and luciferase reporter assay were performed to determine the relationship between miR-26a-5p and CDK6.

Results: MiR-26a-5p was downregulated and CDK6 was upregulated in MSCs-Exo of MCAO and OGD model. MSCs-Exo-miR-26a-5p mimics significantly reduced cell apoptosis of OGD-injured BV-2 cells. MSCs-Exo-miR-26a-5p mimics significantly reduced infarct volume of MCAO-induced mice. Luciferase reporter assay revealed that CDK-6 was a target of miR-26a-5p. In addition, MSCs-Exo-miR-26a-5p mimics significantly decreased the expression of CDK6 in both OGD-induced BV-2 cells and MCAO-treated mice brains.

Conclusion: Our results indicated that MSCs-Exo attenuated I/R injury in mice by inhibiting microglia apoptosis via exosomal miR-26a-5p mediated suppression of CDK6. Our study shed light on the application of MSC-Exo as a potential therapeutic tool for cerebral I/R injury.

\section{Background}

Ischemic stroke is becoming a major leading cause of disability and death worldwide(1). Approximately $85 \%$ of all reported strokes are because of cerebral ischemia that always occurs when an embolus or thrombus blocks the major cerebral artery and then leads to the cell death(2). The primary treatments for ischemic stroke are recanalization therapies, which are proved to replenish nutrients and oxygen, as well as remove toxic metabolites(3). The identification of new therapies or understanding of critical mediators participated in the progression of cerebral ischemic stroke is still urgent.

Exosomes are nano-scale messengers carrying bio-molecular cargo of RNA, DNA, and Proteins, and stem cell secreted exosome has been reported that can regulate various autocrine and paracrine functions to alter cell micro-environment and progression in various human diseases(4). The potential clinical values of exosomes can be attributed to their characteristics including their surface markers and molecular cargoes(5), and their ability to cross the brain-blood barrier (BBB)(6), and their potential functions as mediators of the regenerative responses(7). Recently, stem cell therapy has becoming a promising therapeutic option for some neurological disorders including Stroke, Parkinson's disease, Amyotrophic Lateral Sclerosis and Huntington's disease(8). Because the greater benefit on tissue regeneration and 
repair than the stem cells themselves, stem cell-derived exosomes that are responsible for their therapeutic benefits have begun to be noticed. One of the most popular stem cell-derived exosomes which are widely applied in neurological diseases researches are MSCs-Exo(9, 10). However, the research about MSCs-Exo in cerebral ischemia-reperfusion (I/R) injury is lack and attracted us to pay more attention on it.

MiRNAs are found in both intracellular and extracellular environments, and also detected in exosomes participated cell-cell communication(11). Increasing reports have revealed a large number of exosomederived miRNAs in cerebral ischemic stroke. For example, exosomal miR-223 was significantly upregulated in acute ischemic stroke, and high level of exosomal miR-223 was associated with the occurrence of acute ischemic stroke and stroke severity(12). M2 microglia-derived exosomes has been showed to protect the mouse brain against I/R injury via exosomal miR-124(13). Exosomes-derived miR26a-5p, newly identified exosomal miRNA, has been revealed to play important roles in human diseases. Human bone MSCs-derived exosomes overexpressing miRNA-26a-5p significantly alleviate osteoarthritis through the downregulation of PTGS2(14). Dysregulation of exosomal miRNAs such as miR-26a-5p may affect the regulatory pathways associated with clopidogrel-induced liver injury(15). In addition, one previous study has demonstrated that miR-26a-5p is downregulated in myocardial I/R injury, and overexpression of miR-26a-5p effectively improves viability and inhibits apoptosis in cardiomyocytes upon I/R injury by inhibiting PTEN expression(16). Although exosomal miR-26a-5p play crucial functions in various diseases including $\mathrm{l} / \mathrm{R}$ injury of some organs, its function and molecular mechanism in cerebral I/R injury remains unclear.

CDK6 is known as a classic cell cycle kinase by forming complexes with D-type cyclins, which can phosphorylate the Retinoblastoma protein $(\mathrm{Rb})$ to regulate transition from $\mathrm{G} 1$ to $\mathrm{S}$ phase(17). Previous studies have demonstrated that CDK6 is closely involved in the biological processes including cell proliferation, apoptosis and the transition from $\mathrm{G} 1$ to $S$ phase in cerebral ischemic stroke $(18,19)$. Interestingly, Huang et al. found that miR-26a-5p inhibits the growth of breast cancer cells by downregulating the expression of CDK6(20), suggesting that CDK6 might be a direct or indirect downstream gene. Meanwhile, a miRNA profiling and bioinformatics prediction performed by Canturk et at. indicated that the CDK6 was predicted to be a target of miR-26a-5p, and the axis was significantly associated with the progression of bladder cancer(21), indicating that CDK6 might be a direct target of miR-26a-5p.

In the present study, we explored the role of exosomal miR-26a-5p/CDK6 axis in cerebral I/R injury. Taken together, our study demonstrated that MSCs-derived exosomes overexpressing miR-26a-5p effectively reduced cell apoptosis of OGD-treated microglia cell line BV-2 cells in vitro, and also attenuated MCAOinduced infarct volume in mice, suggesting that exosomal miR-26a-5p might be a potential therapeutic target for cerebral ischemic stroke.

\section{Methods}




\section{Isolation of MSCs-derived Exosomes}

Mesenchymal stem cells (MSCs) were isolated from six adult C57BL/6J mice by the whole bone marrow adherence method as previously described(22). $2 \times 10^{6} \mathrm{MSCs}$ were cultured in $100 \mathrm{~mm}$ dishes, MSCs were washed with PBS and kept in fetal bovine serum (FBS)-free L-DMEM medium (Gibco, CA, USA) for $48 \mathrm{~h}$ when cells reached nearly $80 \sim 90 \%$ confluence. Then the supernatant was collected and subject to sequential centrifugation to obtain the exosomes according to a previous study(23). The precipitated exosomes were stored at $-80^{\circ} \mathrm{C}$.

\section{Characterization of MSCs-derived Exosomes}

For transmission electron microscopy (TEM), isolated exosomes were fixed with $1 \%$ glutaraldehyde, then a drop of fixed exosomes was spotted onto a formvar/carbon-coated grid and negatively stained with $3 \%$ aqueous phosphotungstic acid for $1 \mathrm{~min}$. Subsequently, MSCs-derived exosomes were observed under TEM (Hitachi, Tokyo, Japan, SU-8010). For flow cytometry analysis of exosomes, MSCs-derived exosomes were mixed with $3 \mu \mathrm{m}$ aldehyde/sulfate latex beads (Invitrogen, Batch Num: 979383) for 10 min with continuous rotation. $1 \mathrm{M}$ glycine in PBS containing 2\% BSA was added into the mixture to stop the reaction. Beads coated with exosomes were incubated with antibodies CD63-FITC (Lot: GR320523-9, Abcam), CD81-PE (Cat: MA5-17941, Invitrogen) at $37{ }^{\circ} \mathrm{C}$ for $25 \mathrm{~min}$. Then a FCM flow cytometry (BD FACSalibur) was used to detect the mesenchymal markers.

\section{Animal model of cerebral ischemia-reperfusion (I/R)}

A total of 30 adult C57BL/6J mice (8 weeks old and $250 \mathrm{~g}$ in weight) were provided from Anima Center of Third Military Medical University (Chongqing, China). All animal producers were approved by the Institutional Animal Care and Use Committee of Anima Center of Third Military Medical University (Chongqing,China).. Mice with cerebral ischemia was induced by right middle cerebral artery occlusion (MCAO) as described previously with minor modifications(24). Reperfusion was performed by withdrawing the suture $1 \mathrm{~h}$ after MCAO. For sham group, mice accepted the same operation except MCAO procedure. $2 \mathrm{~h}$ after reperfusion, $200 \mu \mathrm{L} /$ mice MSCs-exosomes-miR-NC or MSCs-exosomes-miR-26a-5p mimics (RiboBio, Guangzhou, China) were immediately injected through the tail vein. The mice in the control group were given an equal volume of normal saline ( $n=6$ in each group). All mice were divided into four groups: Sham group, MCAO group, MCAO + MSCs-exosome-miR-NC, and MCAO + MSCsexosome-miR-26a-5p mimics group.

\section{Determination of infarct size}

After intraperitoneal injection of $3 \%$ sodium pentobarbital, the brains of mice in different groups were removed, placed in 4\% PFA overnight and then fully dehydrated in $30 \%$ sucrose for 2 days. The brain tissue was then cut into coronal sections with approximately $2 \mathrm{~mm}$ in thickness, and then quickly incubated in $2 \%$ TTC solution (Sigma) at $37^{\circ} \mathrm{C}$ for $15 \mathrm{~min}$. Subsequently, the sections were fixed by $4 \%$ paraformaldehyde and photographed under a $\times 100$ optical microscope (Olympus Corporation). The 
image analysis software Image $\mathrm{J} 1.43$ (National Institutes of Health) was used to evaluate the relative infarct percentage according to the following equation: infarct size $=100 \% \times$ (infarcted volume/total brain volume).

\section{MSCs of OGD model}

MSCs was cultured with in Dulbecco's modified Eagle's medium (DMEM, Gibco, USA) supplemented with $10 \%(\mathrm{v} / \mathrm{v}$ ) fetal bovine serum (FBS, Biological Industries, USA), $100 \mathrm{U} / \mathrm{mL}$ penicillin and $100 \mu \mathrm{g} / \mathrm{mL}$ streptomycin (Gibco, USA) at $37^{\circ} \mathrm{C}$ under $5 \% \mathrm{CO}_{2}$. To mimics the ischemia condition in vitro, cells were firstly induced by oxygen-glucose deprivation (OGD) by using deoxygenated glucose-free DMEM medium in an incubator with $95 \% \mathrm{~N}_{2}, 5 \% \mathrm{CO}^{2}$ for 1,2 and $4 \mathrm{~h}$, then cells were transferred into normal condition for an additional $24 \mathrm{~h}$ for re-oxygenation. Cells treated without OGD were used as the control group.

\section{Cell transfection}

MSCs were transfected with $50 \mathrm{nM}$ miR-26a-5p mimics or miR-NC (RiboBio, Guangzhou, China) by using Lipofectamine ${ }^{\circledR} 2000$ Transfection Reagent (Gibco Life Technologies) according to the manufacturer's instructions. The sequences used in this study as follows: miR-26a-5p mimics: $5^{\prime}-$ UUCAAGUAAUCCAGGAUAGGCU-3'; miR-NC: 5'-UUCUCCGAACGUGUCACGUTT-3'. When needed, the exosomes were isolated from MSCs transfected with miR-26a-5p mimics or miR-NC and used for the treatment of microglia cell line BV-2 cells.

\section{The treatment of microglia}

To simulate an in vivo environment of MCAO in microglia, BV-2 cells (Bioleaf, Shanghai, China) were induced by OGD/R treatment as similarly as the MSCs of OGD model above. Then $200 \mu \mathrm{g} / \mathrm{mL}$ MSCsderived exosomes with overexpression of miR-26a-5p mimics or miR-NC were added into the culture medium to explore the effect of MSCs-Exo on microglia function.

\section{CCK-8 assay}

Cell viability was evaluated by using a Cell Counting Kit-8 (CCK-8, Dojindo Molecular Technologies, Gaithersburg, MD). In brief, MSCs were seeded into 96-well plates overnight. After the treatment, $10 \mathrm{ul}$ of CCK-8 reagent was added to each well at $24,48,72$ and $96 \mathrm{~h}$ and then incubated for another $4 \mathrm{~h}$. The absorbance at $450 \mathrm{~nm}$ was detected with a microplate reader. The absorbance was detected at $450 \mathrm{~nm}$ with a microplate reader.

\section{Luciferase reporter assay}

The putative binding sites between miR-26a-5p and 3'-UTR of CDK6 were predicted by starBase (http://starbase.sysu.edu.cn/). The mutant-type (MUT) of 3'-UTR (CDK6- MUT) and wild type (WT) of 3'UTR (CDK6-WT) were amplified and cloned into the pmirGLO dual luciferase reporter vector (Promega, Madison, WI, USA). Then the luciferase reporter plasmids were co-transfected with miR-26a-5p mimics or 
miR-NC into 293T cells by using Lipofectamine ${ }^{\circledR} 2000$ Transfection Reagent. $48 \mathrm{~h}$ fater transfection, cells were lysed and the relative luciferase activity was detected by the dual-luciferase reporter gene assay (Promega).

\section{RNA extraction and qRT-PCR analysis}

Total RNA was extracted from cultured cells or mice brains by using Trizol reagent (Invitrogen). Singlestrand cDNA was synthesized using a universal cDNA synthesis kit (Qiagen, Hilden, Germany) according to the manufacturer's instructions. The expression of targets was tested with a fast real-time PCR system (7900 HT, ABI, Foster City, CA) by using a SYBR Green master mix (Qiagen). The relative expression

change of targets was analyzed by the $2^{-\triangle \triangle C t}$ method with GAPDH and U6 as the internal references. The primers used as follows: miR-26a-5p: forward: 5'-GACGGTACCTTGTCCCTGAATGTAACTCG-3' reverse: 5'GTTCTCGAGAAAGCAGTCCCAGCCTAAA-3'; U6: forward: 5'-CTCGCTTCGGCAGCACA-3', reverse: 5'AACGCTTCACGAATTTGCGT-3'; GAPDH forward: 5'-CAAGGTCATCCATGACAACTTTG-3', reverse: 5'GTCCACCACCCTGTTGCTGTAG-3'.

\section{Western blot}

Total protein of mice brains or cultured cells was isolated by using RIPA lysis buffer (Beyotime Institute of Biotechnology). Approximately equal amounts of protein were separated by $10 \%$ SDS-PAGE and transferred onto PVDF membranes (Millipore). After blocking with 5\% skim milk, the membranes were incubated with primary antibodies including CD9, CD63, CD81, HSP70, CDK6, cleaved Caspase3 (cCaspase3), cleaved PARP, and GAPDH antibodies (diluted into 1:1000; Cell Signaling Technology, USA) overnight at $4^{\circ} \mathrm{C}$. On the next day, the membranes were incubated with HRP-labeled secondary antibody at room temperature for $1 \mathrm{~h}$. After washing with TBS-T, the protein bands were visualized by ECL reagent and band intensity of targets was quantified by Image-Pro Plus 6.0 software (Media Cybernetic).

\section{Apoptosis analysis}

Cell apoptosis was analyzed by using a commercialized Annexin V-FITC/PI Apoptosis Detection Kit (YEASEN, Shanghai) according to the manufacturer's protocols. Briefly, BV-2 cells were washed with PBS, and re-suspended in $100 \mathrm{ul}$ of binding buffer, and $5 \mathrm{ul}$ of Annexin V-FITC and $10 \mathrm{ul}$ of propidium iodide (PI) were gently mixed and added into cell suspension, then incubated in the dark for 15 min. Finally, cell apoptosis was detected by flow cytometry (BD FACS Canto II, USA).

\section{In situ detection of fragmented DNA (TUNEL assay)}

Brains tissues were collected and the apoptosis in vivo was evaluated TUNEL staining kit (YEASEN, Shanghai) as the manufacturer's instructions. TUNEL positive brain cells were counted under a fluorescence microscope. Specially, cell nucleus dyed green were considered to be apoptotic cells, and the rate of apoptosis (\%) was calculated as the percentage of TUNEL positive cell nucleus in 5 random fields for each sample. 


\section{Statistical analysis}

All data were presented as mean $\pm S D$, and each experiment was repeated three times. Statistical analysis was performed by using GraphPad Prism 6.0 software. Groups comparison was performed with two tailed Student's $t$ test (two groups) or one-way analysis of variance (ANOVA; multiple groups). $P<0.05$ was considered to be significant.

\section{Results}

\section{Characterization of MSCs-derived exosomes}

To ensure the reliability of subsequent experiments, we firstly detected the characterization of MSCs-derived exosomes. Under the transmission electronic microscope (TCM), MSCs-derived exosomes showed widespread, derangement distribution, and conglobation in some areas, and the vesicles exhibited typical exosome morphology with a dimension varying from 30 to $150 \mathrm{~nm}$ (Fig. 1A). Flow cytometry indicated that CD63 and CD81 positive rates were $30.84 \%$ and $43.75 \%$, respectively (Fig. 1B), suggesting that MSCs-exosomes isolated were of certain purity. Meanwhile, the expression of exosomal markers was evaluated by western blot, and the results showed that the exosomal markers including CD9, CD63, CD81 and HSP70 were all expressed in MSCs and MSCs-derived exosomes, and their levels in MSCs-derived exosomes were higher than that in MSCs (Fig. 1C). These results suggested that the isolated exosomes could be better used for the subsequent experiments.

\section{MiR-26a-5p was downregulated and CDK6 was upregulated in MSCs-derived exosomes of MCAO and OGD model}

Next, we explore the expression of miR-26a-5p and CDK6 in exosomes of cerebral I/R in vitro and in vivo. Compared with sham group, the expression of miR-26a-5p was significantly downregulated both in MSCs-derived exosomes ( $p<0.01$, Fig. $2 A$ ) and brains tissues ( $p<0.01$, Fig. $2 B$ ) of MCAO-induced mice. Similarly, the level of mIR-26a-5p in the exosomes of OGD-induced MSCs was also downregulated compared with control group (without OGD treatment) in a time dependent manner ( $<<0.05$, Fig. $2 \mathrm{C}$ ). In addition, the protein level of CDK6 was increased in MSCs-derived exosomes of MCAO-induced mice compared with that of sham group ( $p<0.01$, Fig. 2D). Meanwhile, the expression of CDK6 was also upregulated in the exosomes of OGD-induced MSCs compared with control group ( $p<0.05$, Fig. $2 E)$. These results revealed that miR-26a-5p was downregulated and CDK6 was upregulated in MSCs-derived exosomes of MCAO and OGD model.

\section{MiR-26a-5p mimics reversed the effects of MSCs-derived exosomes in reducing cell apoptosis of OGD- injured microglia}

To further explore the effect of exosomal miR-26a-5p on microglia function, BV-2 cells were treated OGD for the given time, and then treated with $200 \mu \mathrm{g} / \mathrm{mL}$ MSCs-Exo-miR-26a-5p mimics or MSCs-Exo-miR-NC. CCK-8 assay indicated that OGD treatment significantly reduced cell viability compared with control 
group ( $\mathrm{p}<0.01$ ), and OGD-treated BV-2 cells cultured with MSCs-Exo-miR-26a-5p showed greater cell viability than MSC-Exo-miR-NC group $(p<0.05$, Fig. 3A). Meanwhile, OGD treatment significantly exacerbated the apoptosis rate of BV-2 cells compared with control group $(p<0.001)$, and the elevated apoptosis of BV-2 cells stimulated by OGD were obviously reduced by MSCs-Exos-miR-26a-5p mimics compared with MSCs-Exo-miR-NC group ( $p<0.01$ ) (Fig. 3B). In addition, the expression of apoptosisrelated proteins was evaluated by western blot, and the results showed that the expression of c-caspase 3 and c-PARP was increased in BV-2 cells after OGD treatment compared with control group $(p<0.001)$, and the effect was significantly reversed by MSCs-Exo-miR-26a-5p mimics compared with MSCs-ExomiR-NC group $(p<0.05)$ (Fig. 3C). These results indicated that overexpression of miR-26a-5p could reverse the effects of MSCs-derived exosomes in reducing cell apoptosis of OGD-injured microglia.

\section{MSCs-Exo-miR-26a-5p mimic attenuated ischemia-reperfusion injury in MCAO/R model}

We then explore the neuroprotective effect of MSCs-Exo-miR-26a-5p on I/R injury in vivo through injection with $200 \mu \mathrm{L} /$ mice MSCs-exosomes-miR-NC or MSCs-exosomes-miR-26a-5p mimics by the tail vein after 2 $\mathrm{h}$ for MCAO/R treatment. The expression of miR-26a-5p in brain tissues was firstly detected by qRT-PCR and the results showed that MCAO/R treatment significantly reduced miR-26a-5p level compared with sham operation ( $<$ 0.001), and compared with MSCs-Exo-miR-NC group, MSCs-Exo-miR-26a-5p mimics obviously attenuated MCAO/R-induced decrease of miR-26a-5p $(p<0.01)$ (Fig. 4A). The results of TTC staining (Fig. 4B) showed that MCAO/R treatment significantly enhanced infarct volume compared with sham operation ( $p$ 0.001), and compared with Exo-miR-NC group, Exo-miR-26a-5p mimics markedly reduced infarct percentage $(p<0.01)$. Meanwhile, cell apoptosis in brain tissues was determined by TUNEL staining and the results indicated that TUNEL positive cells were increased after MCAO/R treatment compared with sham operation $(p<0.001)$, and Exo-miR-26a-5p mimics obviously reduced TUNEL positive cells of rat brains after MCAO/R treatment compared with Exo-miR-NC group $(p<0.01)$ (Fig. 4C). In addition, the expression of apoptosis-related proteins in brain tissues was also evaluated by western blot and the results showed that MCAO/R-treatment significantly increased the expression of ccaspase 3 and c-PARP compared with sham operation, and compared with Exo-miR-NC group, Exo-miR26a-5p mimics obviously reduced the level of c-caspase 3 and c-PARP in rat brains induced by MCAO/R. These results suggested that MSCs-Exo-miR-26a-5p mimics attenuated ischemia-reperfusion injury in MCAO/R model in vivo.

\section{The effect of miR-26a-5p was partially mediated by CDK6}

To further determine the molecular mechanism of miR-26a-5p, we analyzed the sequence of the 3'-UTR of the human CDK6 gene. We found that there was a putative binding site between miR-26a-5p and 3'-UTR of CDK6 by using Targetscan software (http://www.targetscan.org) (Fig. 5A). Then miR-26a-5p mimics or miR-NC was transfected into 293T cells and qRT-PCR showed that miR-26a-5p mimics significantly increased the miR-26a-5p level compared with miR-NC ( $<<0.01$, Fig. 5B). Meanwhile, luciferase reporter assay indicated that miR-26a-5p mimics obviously reduced the relative luciferase activity of CDK6-WT compared with miR-NC ( $<$ 0.01), while had no change on CDK6-MUT (Fig. 5C). Next, miR-26a-5p mimics 
or miR-NC was transfected into BV-2 cells and we found that miR-26a-5p mimics significantly decreased the expression of CDK6 in BV-2 cells at both mRNA level $(p<0.01)$ and protein level $(p<0.01)$ compared with miR-NC (Fig. 5D and E). Moreover, we found that OGD/R treatment significantly increased CDK6 level compared with control group ( $<$ 0.01), and Exo-miR-26a-5p mimics obviously reduced CDK6 level in BV2 cells compared with Exo-miR-NC group $(p<0.05)$ (Fig. $5 F$ ). In addition, MCAO/R treatment significantly increased CDK6 level in mice brains compared with sham operation $(p<0.01)$, and Exo-miR-26a-5p mimics markedly reduced CDK6 level in mice brains induced by MCAO/R treatment compared with ExomiR-NC group $(p<0.05)$ (Fig. 5G). These data suggested that MSCs-derived exosomes with miR-26a-5p overexpression attenuated I/R brain injury through targeting CDK6.

\section{Discussion}

In this study, we explore the role of exosomal miR-26a-5p in cerebral I/R injury, and found that miR-26a-5p was downregulated in MSCs-derived exosomes of MCAO and OGD model. Moreover, MSCs-Exo-miR-26a$5 p$ mimics effectively reduced cell apoptosis of BV-2 cells submitted to OGD/R treatment, and also reduced infarct volume

of mice submitted to MCAO/R treatment by elevating the expression of CDK6. Our results provided that MSCs-Exo overexpressing miR-26a-5p could effectively attenuate l/R brain injury through targeting CDK6.

A series of miRNAs have been found to be loaded by exosomes of different types of cells, and have been showed to play crucial roles in neuron injury(25). For example, miRNA-181a overexpression in MSCsderived exosomes suppress inflammatory response after myocardial I/R injury(26). Cortical neuronderived exosomal miRNA-181c-3p inhibits neuro-inflammation by downregulating CXCL1 in astrocytes of a rat model with ischemic brain injury(27). Exosomes derived from miR-138-5p-overexpressing bone marrow-derived mesenchymal stem cells confer neuroprotection to astrocytes following ischemic stroke via inhibition of LCN2(25). Exosome-shuttled miR-92b-3p from ischemic preconditioned astrocytes protects neurons against oxygen and glucose deprivation(28). Exosomal miR-26a-5p level has been identified to be decreased in hUCMSCs, and exosomal miR-26b-5p from hUCMSCs could repress M1 polarization of microglia by targeting $\mathrm{CH} 25 \mathrm{H}$ to inactivate the TLR pathway, then finally relieve nerve injury after cerebral I/R(29). In this study, we found that miR-26a-5p was downregulated in MSCs-derived exosomes of MCAO and OGD model. To explore the effect of exosomal miR-26a-5p in cerebral I/R injury, miR-26a-5p overexpressing exosomes were isolated from MSCs and treated with BV-2 cells or injected with mice submitted MCAO treatment. Moreover, MSCs-Exo-miR-26a-5p mimics effectively reduced cell apoptosis of BV-2 cells submitted to OGD/R treatment, and also reduced infarct volume of mice submitted to MCAO/R treatment. These results indicated the protective effect of exosomal miR-25a-5p in cerebral I/R injury, which might be regarded as a novel therapeutic target for cerebral ischemic stroke.

Here, we found that CDK6 was upregulated in MSCs-derived exosomes of MCAO and OGD model, suggesting the essential role of CDK6 in cerebral I/R injury. Previous studies have reported that CDK6 could serve as a target of miRNAs to participate in neuron injury. MiR-99a overexpression inhibits $\mathrm{H}_{2} \mathrm{O}_{2}$ 
induced G1/S phase transition along with a significant low level of CDK6 in neuro-2a cells(30). MiR-424 has been identified to protects from permanent focal cerebral ischemia injury in mice through targeting CDK6 to inhibit microglia activation (31). However, the regulatory network of CDK6 involved in exosomal miRNAs has not been well studied. cerebral I/R injury, bioinformatics prediction was performed and suggested that CDK6 might a direct target of exosomal miR-26a-5p. Then luciferase reporter assay further confirmed the correlation between exosome miR-26a-5- and CDK6. In addition, MSCs-Exo-miR-26a$5 p$ mimics obviously reduced CDK6 level in BV-2 cells after OGD/R treatment, and also reduced CDK6 level in mice brains induced by MCAO/R treatment. All these data suggested that the protective effect of MSCs-Exo-miR-26a-5p mimics on I/R injury both in vitro and in vivo might be mediated by CDK6, providing a new highlight of exosomal miRNAs and cell proliferation-related proteins involved in cerebral I/R injury. Our study suggests that EMSCs-derived exosomes overexpressing miR-26a-5p might be applied for the personalized treatment against cerebral ischemic stroke.

However, there was a limitation existing in the study which was determined in the subsequent experiments. That was, whether high level of CDK6 reversed the protective effect of MSCs-Exo-miR-26a$5 p$ mimics on cerebral I/R injury.

\section{Conclusion}

In summary, our results demonstrated that MSCs-derived exosomes could effectively attenuate I/R injury in vivo and inhibit microglia apoptosis in vitro by exosomal miR-26a-5p mediated downregulation of CDK6, suggesting that miR-26a-5p might be a novel therapeutic target for cerebral I/R injury.

\section{Declarations}

\section{Ethics approval and consent to participate}

Not applicable.

\section{Consent for publication}

All authors give the consent for publication

\section{Acknowledgements}

Not applicable.

\section{Funding}

This work was supported by the National Natural Science Foundation of China (Grant No. 81571129).

\section{Availability of data and materials}


Any additional information related to this study is available from the author for correspondence upon reasonable request.

\section{Authors' contributions}

WH conceived and designed the experiments. CC, XC, YW, WC, XZ and WT performed the experiments. CC analyzed the data and wrote the manuscript. All authors read and approved the final manuscrip

\section{Competing interests}

The authors declare that they have no competing interests

\section{References}

1. Barthels D, Das H. (2020) Current advances in ischemic stroke research and therapies. Biochimica et biophysica acta. Molecular basis of disease 1866: 165260.

2. Powers WJ. (2020) Acute Ischemic Stroke. N Engl J Med 383: 252-260.

3. Phipps MS, Cronin CA. (2020) Management of acute ischemic stroke. BMJ (Clinical research ed.) 368: 16983.

4. Sharma A. (2018) Role of stem cell derived exosomes in tumor biology. International journal of cancer 142: 1086-1092.

5. Dorayappan KDP, Wallbillich JJ, Cohn DE, Selvendiran K. (2016) The biological significance and clinical applications of exosomes in ovarian cancer. Gynecologic oncology 142: 199-205.

6. Kalani A, Tyagi A, Tyagi N. (2014) Exosomes: mediators of neurodegeneration, neuroprotection and therapeutics. Molecular neurobiology 49: 590-600.

7. Xin $\mathrm{H}$, et al. (2012) Exosome-mediated transfer of miR-133b from multipotent mesenchymal stromal cells to neural cells contributes to neurite outgrowth. Stem cells (Dayton, Ohio) 30: 1556-1564.

8. Yoo J, Kim HS, Hwang DY. (2013) Stem cells as promising therapeutic options for neurological disorders. Journal of cellular biochemistry 114: 743-753.

9. Luarte A, Bátiz LF, Wyneken U, Lafourcade C. (2016) Potential Therapies by Stem Cell-Derived Exosomes in CNS Diseases: Focusing on the Neurogenic Niche. Stem cells international 2016: 5736059.

10. Marote A, Teixeira FG, Mendes-Pinheiro B, Salgado AJ. (2016) MSCs-Derived Exosomes: CellSecreted Nanovesicles with Regenerative Potential. Frontiers in pharmacology 7: 231.

11. Zhang J, et al. (2015) Exosome and exosomal microRNA: trafficking, sorting, and function. Genomics, proteomics \& bioinformatics 13: 17-24.

12. Chen $Y$, et al. (2017) Increased Circulating Exosomal miRNA-223 Is Associated with Acute Ischemic Stroke. Frontiers in neurology 8: 57. 
13. Song Y, et al. (2019) M2 microglia-derived exosomes protect the mouse brain from ischemiareperfusion injury via exosomal miR-124. Theranostics 9: 2910-2923.

14. Jin Z, Ren J, Qi S. (2020) Human bone mesenchymal stem cells-derived exosomes overexpressing microRNA-26a-5p alleviate osteoarthritis via down-regulation of PTGS2. International immunopharmacology 78: 105946.

15. de Freitas RCC, et al. (2017) Modulation of miR-26a-5p and miR-15b-5p Exosomal Expression Associated with Clopidogrel-Induced Hepatotoxicity in HepG2 Cells. Frontiers in pharmacology 8 : 906.

16. Xing X, Guo S. (2020) miR-26a-5p protects against myocardial ischemia/reperfusion injury by regulating the PTEN/PI3K/AKT signaling pathway 53: e9106.

17. Tigan AS, Bellutti F, Kollmann K, Tebb G, SexI V. (2016) CDK6-a review of the past and a glimpse into the future: from cell-cycle control to transcriptional regulation. Oncogene 35: 3083-3091.

18. Li G, et al. (2018) Diagnostic and Immunosuppressive Potential of Elevated Mir-424 Levels in Circulating Immune Cells of Ischemic Stroke Patients. Aging and disease 9: 172-181.

19. Demyanenko S, Uzdensky A. (2017) Profiling of Signaling Proteins in Penumbra After Focal Photothrombotic Infarct in the Rat Brain Cortex. Molecular neurobiology 54: 6839-6856.

20. Huang ZM, et al. (2019) MicroRNA-26a-5p inhibits breast cancer cell growth by suppressing RNF6 expression. The Kaohsiung journal of medical sciences 35: 467-473.

21. Canturk KM, et al. (2014) Investigation of key miRNAs and target genes in bladder cancer using miRNA profiling and bioinformatic tools. Molecular biology reports 41: 8127-8135.

22. Maria AT, et al. (2016) Human adipose mesenchymal stem cells as potent anti-fibrosis therapy for systemic sclerosis. Journal of autoimmunity 70: 31-39.

23. Théry C, Amigorena S, Raposo G, Clayton A. (2006) Isolation and characterization of exosomes from cell culture supernatants and biological fluids. Current protocols in cell biology Chapter 3: Unit 3.22.

24. Yang G, et al. (1994) Human copper-zinc superoxide dismutase transgenic mice are highly resistant to reperfusion injury after focal cerebral ischemia. Stroke 25: 165-170.

25. Deng $Y$, et al. (2019) Exosomes derived from microRNA-138-5p-overexpressing bone marrow-derived mesenchymal stem cells confer neuroprotection to astrocytes following ischemic stroke via inhibition of LCN2. Journal of biological engineering 13: 71.

26. Wei Z, et al. (2019) miRNA-181a over-expression in mesenchymal stem cell-derived exosomes influenced inflammatory response after myocardial ischemia-reperfusion injury. Life sciences 232: 116632.

27. Song H, et al. (2019) Cortical Neuron-Derived Exosomal MicroRNA-181c-3p Inhibits Neuroinflammation by Downregulating CXCL1 in Astrocytes of a Rat Model with Ischemic Brain Injury. Neuroimmunomodulation 26: 217-233.

28. Xu L, et al. (2019) Exosome-shuttled miR-92b-3p from ischemic preconditioned astrocytes protects neurons against oxygen and glucose deprivation. Brain research 1717: 66-73. 
29. Li G, et al. (2020) Exosomes-carried microRNA-26b-5p regulates microglia M1 polarization after cerebral ischemia/reperfusion. Cell cycle (Georgetown, Tex.) 19: 1022-1035.

30. Tao Z, et al. (2015) Neuroprotective effect of microRNA-99a against focal cerebral ischemiareperfusion injury in mice. Journal of the neurological sciences 355: 113-119.

31. Zhao H, et al. (2013) MiRNA-424 protects against permanent focal cerebral ischemia injury in mice involving suppressing microglia activation. Stroke 44: 1706-1713.

\section{Figures}
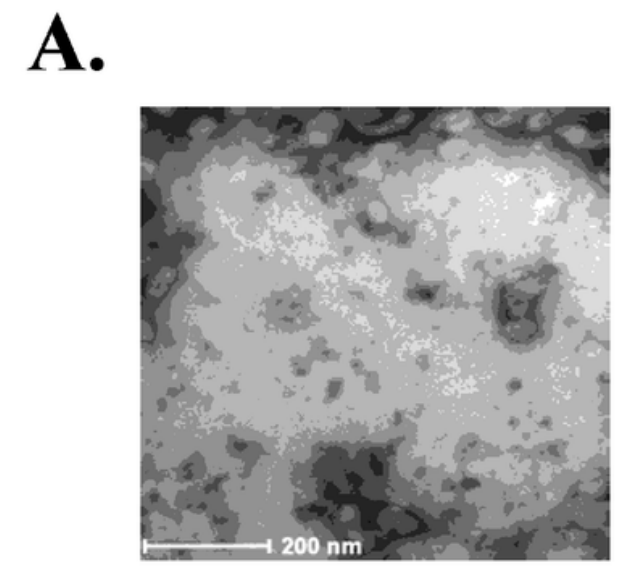

C.
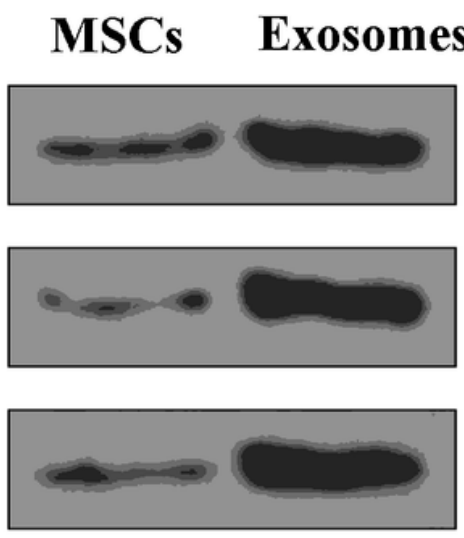

CD9

CD63

\section{CD81}

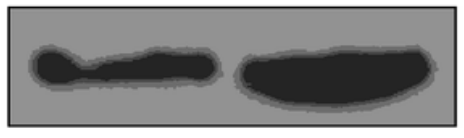

\section{HSP70}
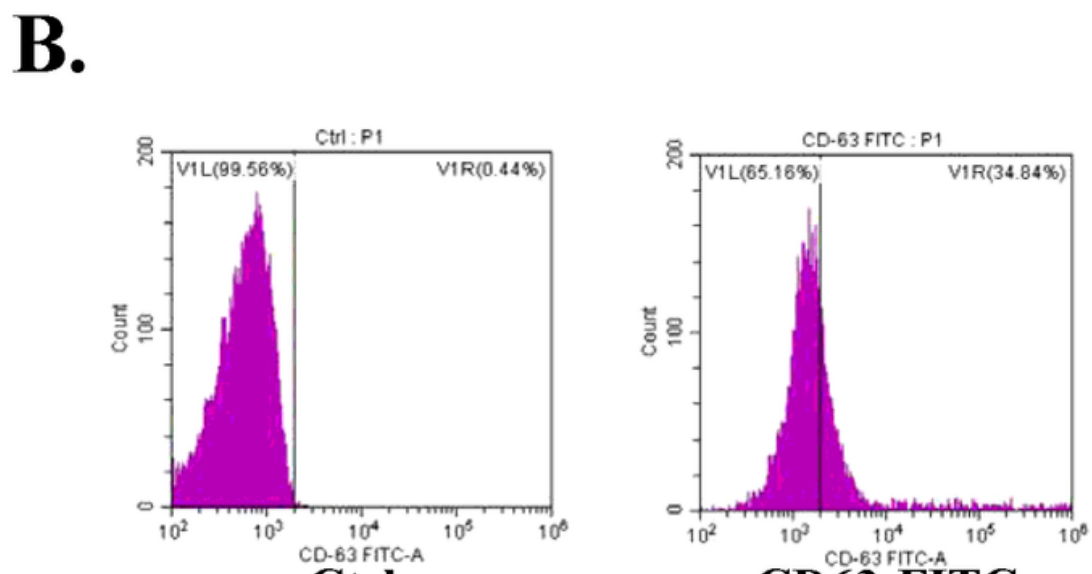

CD63-FITC

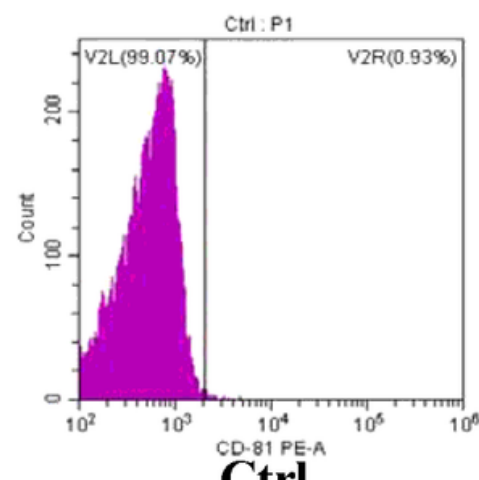

Ctrl

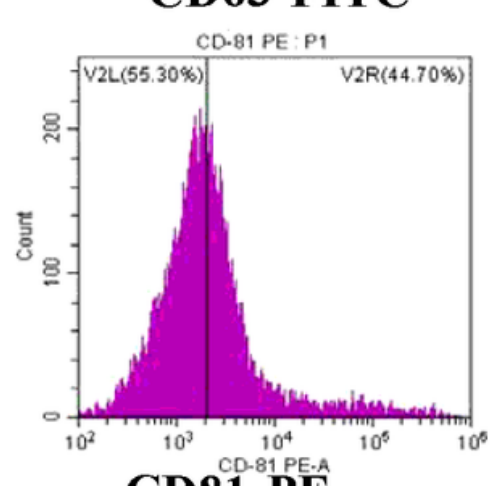

CD81-PE

CD81-PE

\section{Figure 1}

Characterization of MSCs-derived exosomes. (A) Morphological change of MSCs-derived exosomes by TEM. (B) FACS analysis of exosome surface markers CD63 and CD81 in MSCs-derived exosomes. (C) The protein expression of exosome surface markers (CD9, CD63, CD81, and HSP70) in MSCs and MSCsderived exosomes was evaluated by western blot. $\mathrm{N}=3$. 


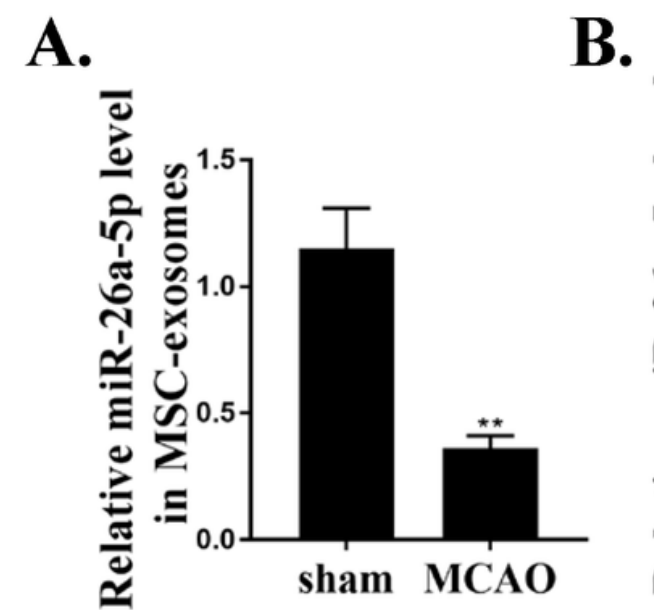

D.
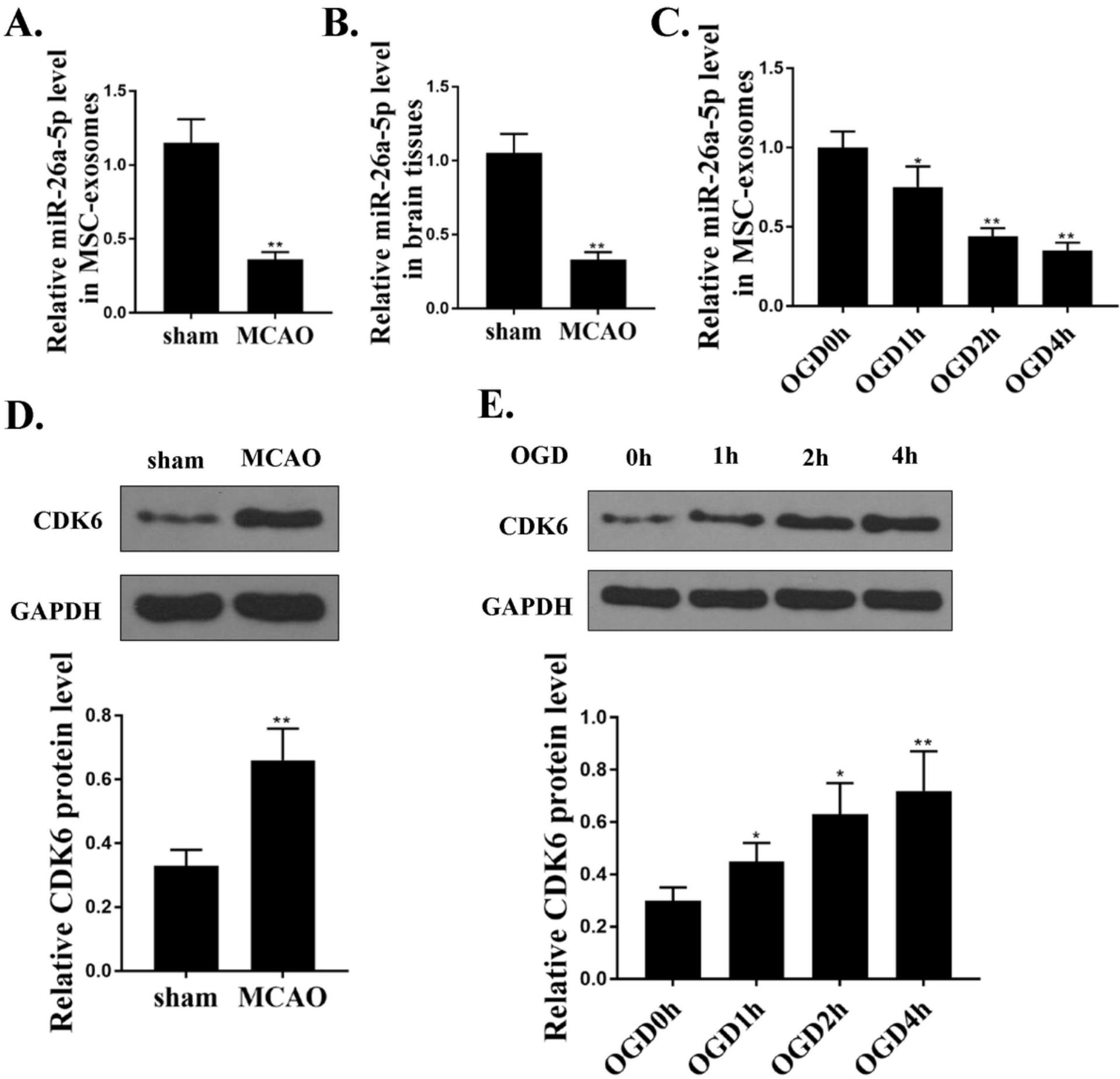

Figure 2

MiR-26a-5p was downregulated and CDK6 was upregulated in MSCs-derived exosomes of MCAO and OGD model. (A and B) The mRNA expression of miR-26a-5p in MSCs-derived exosomes (A) and brain tissues (B) of MCAO-induced mice was evaluated by qRT-PCR $(n=6)$. (C) The level of miR-26a-5p in exosomes of OGD-treated MSCs at indicated time was evaluated by qRT-PCR $(n=3)$. (D) The protein expression of CDK6 in MSCs-derived exosomes of MCAO-induced mice was evaluated by western blot ( $\mathrm{n}$ = 6). (E) The protein expression of CDK6 in exosomes of OGD-treated MSCs at indicated time was evaluated by western blot $(n=3) .{ }^{*} p<0.05,{ }^{\star *} p<0.01$. 
A.

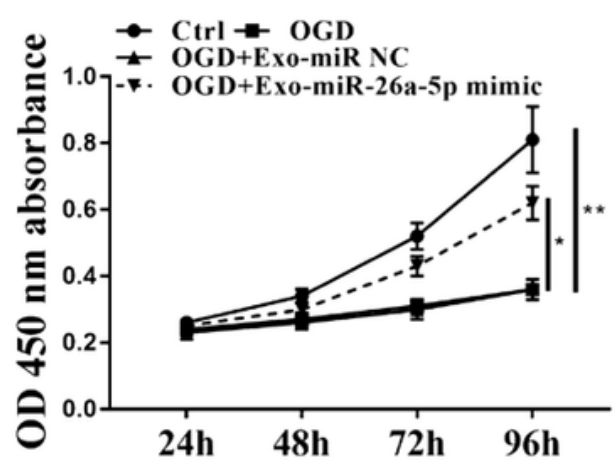

B.
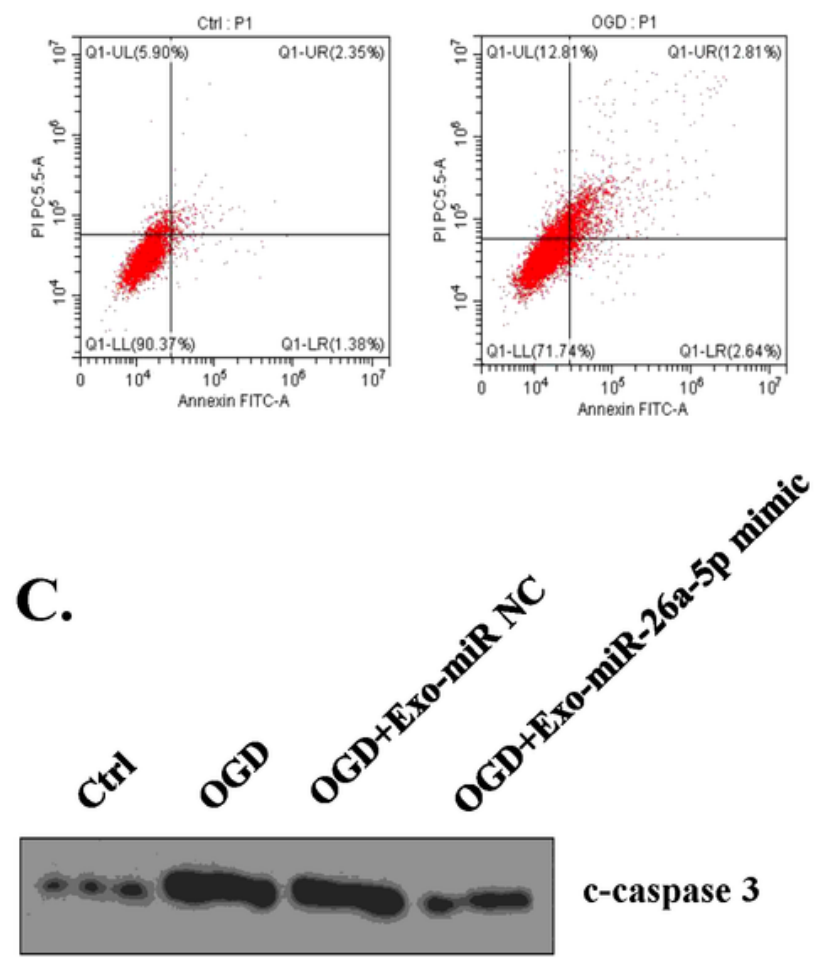

c-caspase 3

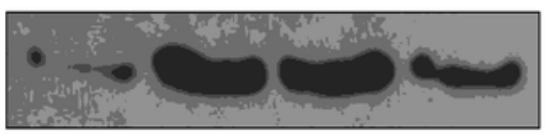

c-PARP

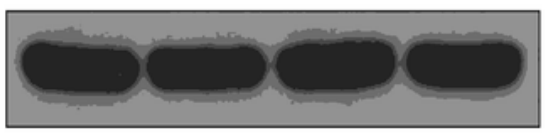

GAPDH

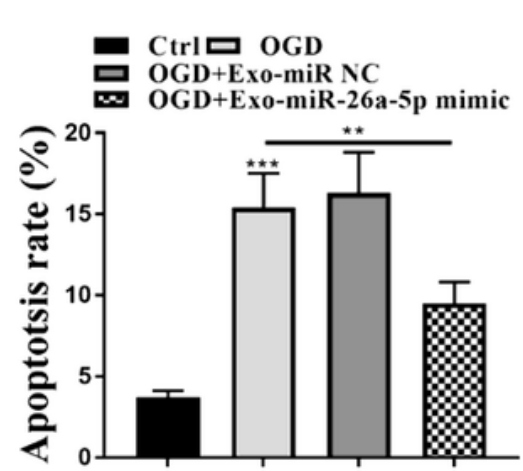

\section{OGD+Exo-miR NC}

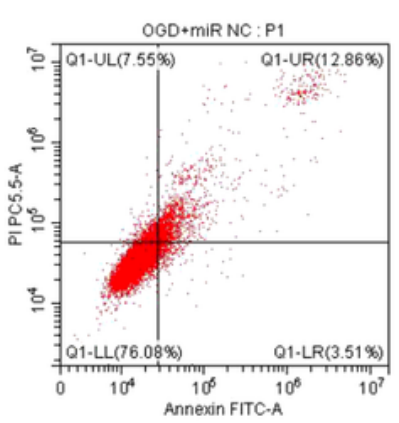

OGD+Exo-miR-

26a-5p mimic
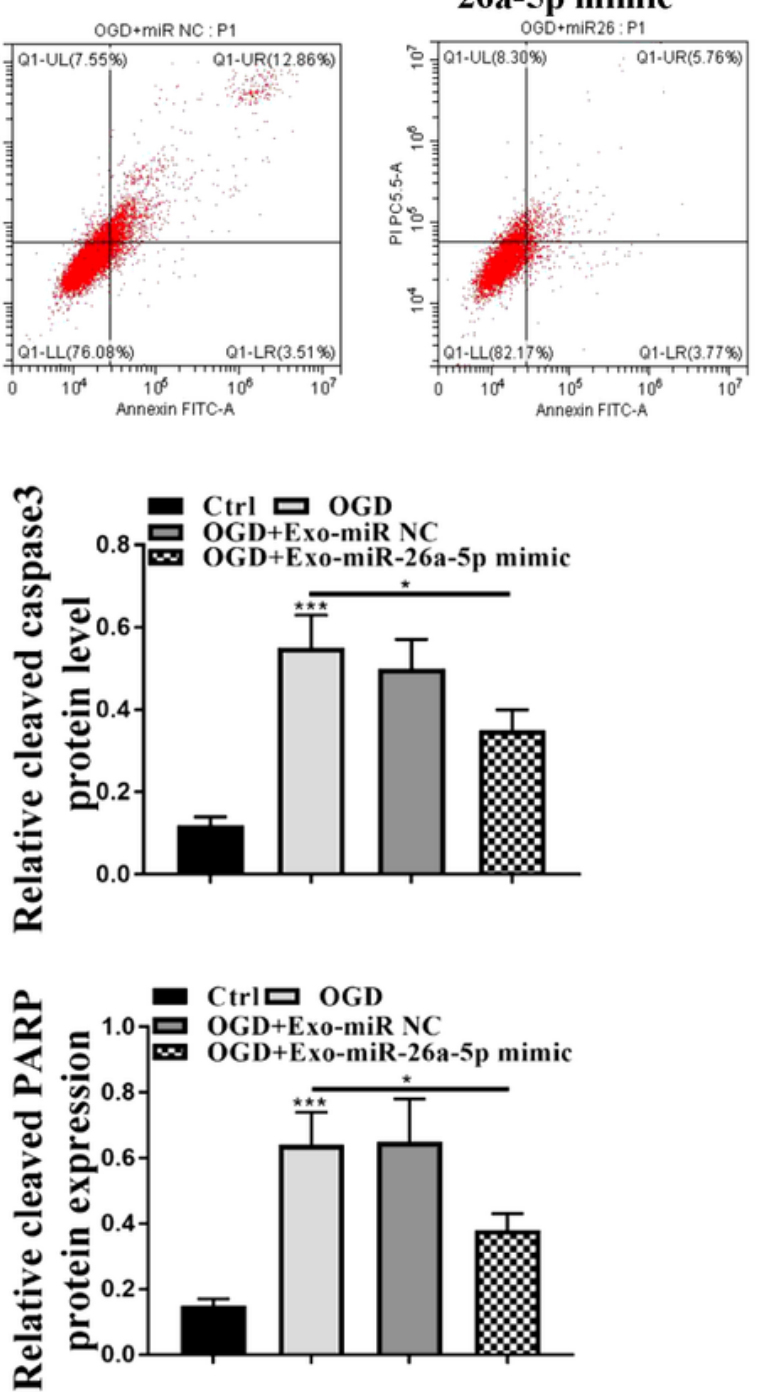

\section{Figure 3}

MiR-26a-5p reversed the effects of MSCs-derived exosomes in reducing cell apoptosis of OGD-injured microglia. BV-2 cells were treated OGD for the given time, and then treated with $200 \mu \mathrm{g} / \mathrm{mL}$ Exo-miR-26a$5 p$ mimics or Exo-miR-NC. (A) Cell viability was evaluated by CCK-8 assay. (B) Cell apoptosis was detected by flow cytometry. (C) The protein expression of apoptosis-related makers was evaluated by western blot. $\mathrm{N}=3 .{ }^{*} \mathrm{p}<0.05,{ }^{*} \mathrm{p}<0.01, * \star * \mathrm{p}<0.001$. 


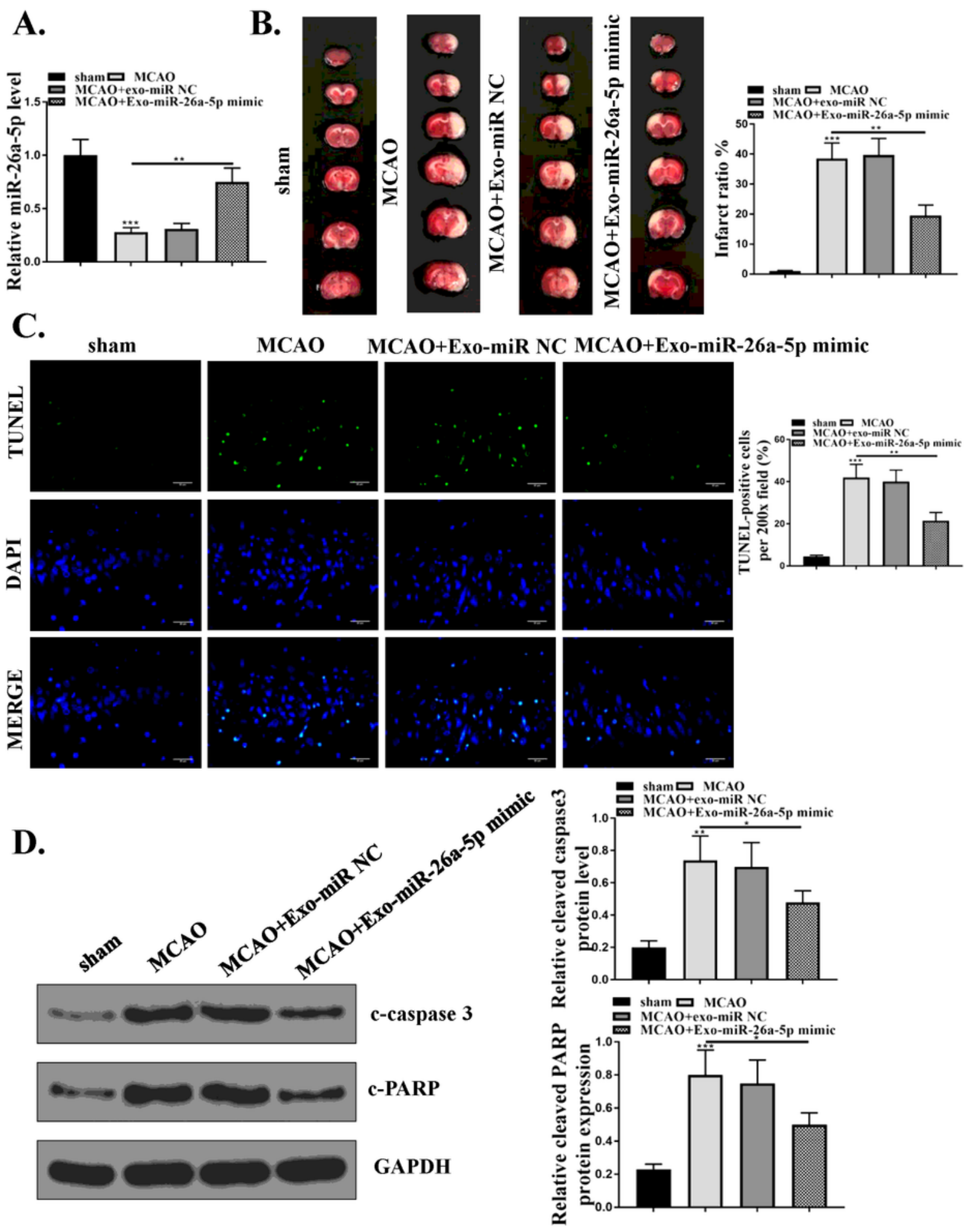

Figure 4

MSCs-Exo-miR-26a-5p mimic attenuated ischemia-reperfusion injury in MCAO/R model. Mice were treated with MCAO/R, and injected with $200 \mu \mathrm{L} /$ mice MSCs-exosomes-miR-NC or MSCs-exosomes-miR26a-5p mimics. (A) The mRNA level of miR-26a-5p in brain tissues was evaluated qRT-PCR. (B) Representative brain sections of I/R induced mice were detected by TTC staining. (C) TUNEL staining in 
brain tissues. (D) The protein level of apoptosis-related markers in brain tissues was detected by western blot. $\mathrm{N}=6,{ }^{*} \mathrm{p}<0.05, * * \mathrm{p}<0.01$, *** $\mathrm{p}<0.001$.

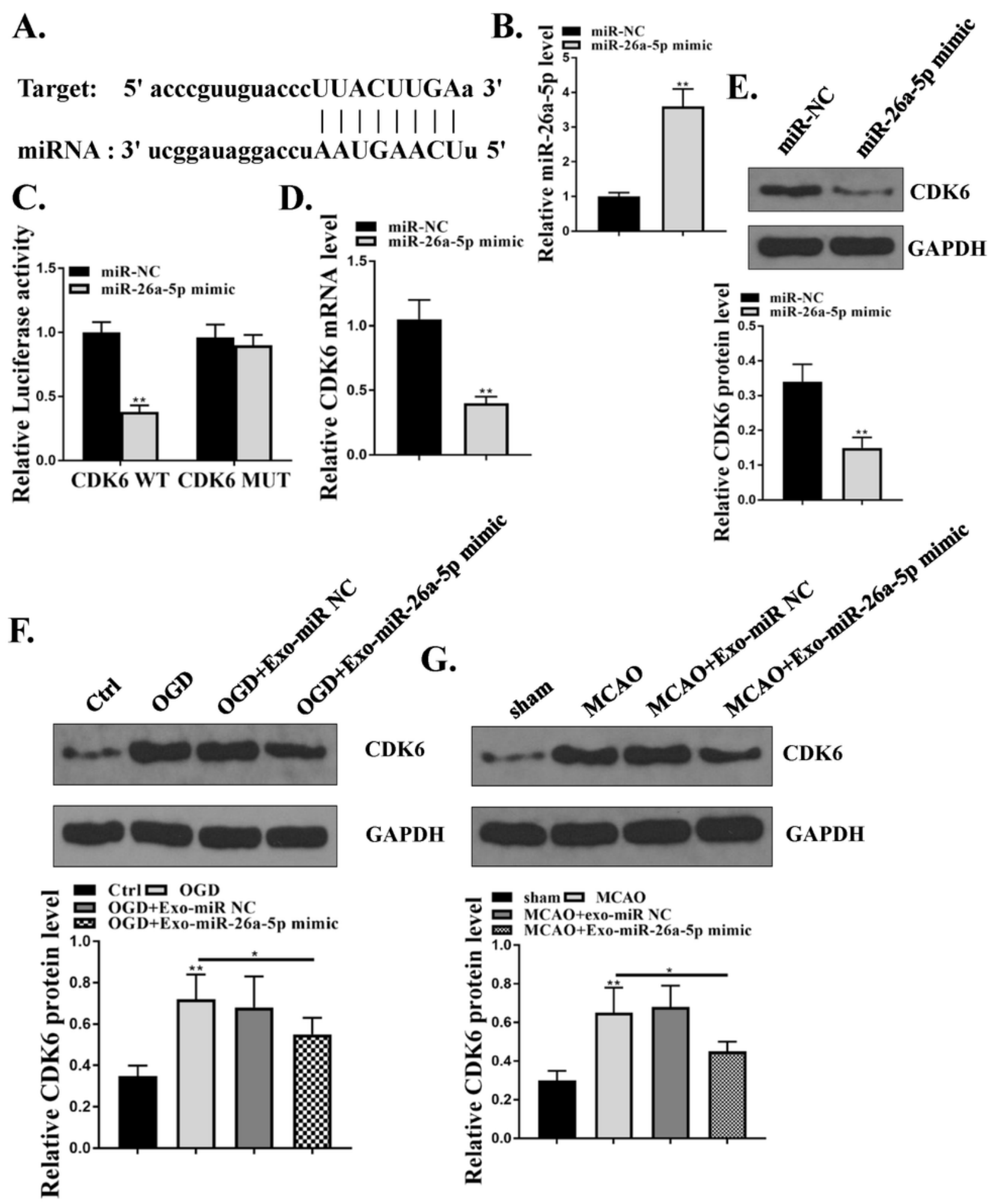

Figure 5

The effect of miR-26a-5p was partially mediated by CDK6. (A) The putative binding site between miR-26a$5 p$ and CDK6 was predicted by Targetscan. (B) 293T cells were transfected with miR-26a-5p mimics or miR-NC, and the mRNA level of miR-26a-5p was detected by qRT-PCR. (C) 293T cells were co-transfected 
with miR-26a-5p mimics or miR-NC and Luc-CDK6-WT or Luc-CDK6-MUT, and the relative luciferase reporter activity was evaluated by dual luciferase reporter system. (D and E) BV-2 cells were transfected with miR-26a-5p or miR-NC, and the expression of CDK6 was detected by qRT-PCR (D) and western blot (E). (F) BV-2 cells were treated by OGD/R, and then treated with $200 \mu \mathrm{g} / \mathrm{mL}$ Exo-miR-26a-5p mimics or Exo-miR-NC, and the protein level of CDK6 was evaluated by western blot. (G) Mice were induced by $\mathrm{MCAO} / \mathrm{R}$, and then injected with $200 \mu \mathrm{L} / \mathrm{mice}$ Exo-miR-NC or Exo-miR-26a-5p mimics. The protein level of CDK6 in brain tissues was detected by western blot. $N=6 .{ }^{*} p<0.05 * * p<0.01$. 